\title{
DISCUSIONES TEÓRICAS CONTEMPORÁNEAS SOBRE POBREZA CAPACIDADES, BIENESTAR Y NECESIDADES HUMANAS
}

Diego Amarilla

\section{Resumen}

En la literatura especializada se manifiesta un amplio consenso en definir a la pobreza desde una perspectiva multidimensional, siendo el centro de la discusión las formas en que se mide el fenómeno. Es en este sentido que el presente artículo ordena cronológicamente las principales vertientes teóricas que nutren la conceptualización contemporánea de la pobreza (el enfoque de los funcionamientos y capacidades y la teoría de las necesidades humanas], destaca las taxonomías elaboradas por los principales referentes teóricos y arriba a conclusiones que invitan a reflexionar sobre los contextos sociohistóricos que determinaron esta producción intelectual y su contribución en la elaboración de un concepto de pobreza multidimensional.

Palabras clave: pobreza multidimensional, capacidades, funcionamientos, necesidades humanas.

\begin{abstract}
Contemporary Theoretical Discussions About Poverty. Capabilities, well-being and human needs In the specialized literature there is a broad consensus in defining poverty from a multidimensional perspective, being the ways in which the phenomenon is measured the center of the discussion. It is in this sense that the present article chronologically ordered the main theoretical aspects that nourish the contemporary conceptualization of poverty (the approach of functionings and capabilities and the theory of human needs], highlighting the taxonomies elaborated by its main theoretical references and arriving at conclusions that invite reflection on the socio-historical contexts that determined this intellectual production and its contribution to the elaboration of a multidimensional concept of poverty.
\end{abstract}

Keywords: multidimensional poverty, capabilities, functionings, human needs.

Diego Amarilla: Maestrando en Sociología y licenciado en Sociología por la Facultad de Ciencias Sociales (UDELAR). Profesor ayudante del Departamento de Sociología de la misma facultad y universidad.

ORCID iD: 0000-0002-2025-011?

Email: amarilladiego7@gmail.com

Recibido: 3 de julio de 2020

Aprobado: 22 de setiembre de 2020 


\section{Introducción}

El término pobreza se ha convertido de acuerdo con sus usos en un significante portador de múltiples significados. Desde el conocimiento vulgar hasta el científico, transitando por la política partidaria y ubicándose de manera habitual en la agenda pública de muchos países, la pobreza se ha transformado en una noción en búsqueda de teoría, tal como lo propone Tabaré Fernández (2010). Esto se da en la medida en que la implementación de estudios e investigaciones en la temática por parte de las ciencias sociales y los organismos internacionales de desarrollo ha propiciado una profunda discusión sobre el alcance y las consecuencias de la pobreza. Es así que Minor Mora (2010) afirma que:

“[...] Dos parecen ser los temas que concitan las disputas principales entre los científicos sociales que concentran su quehacer en torno a esta problemática. Por un lado, la propia definición del objeto: la pobreza — qué es ser pobre-; por otro, la estrategia de medición que debe adoptarse - el método-." (Ringen, citado en Mora, 2010, p. 2).

Aunque las discusiones centrales parecen seguir pasando por las formas unidimensionales y multidimensionales de medición, en la dimensión conceptual existe un amplio consenso sobre la multidimensionalidad de la pobreza (Boltvinik, 2001; Fernández, 2010). En la definición de la pobreza se intersectan diversos clivajes que son de utilidad clave para balizar un camino teórico propenso a caer en eclecticismos. Por esto, se trae a colación la distinción realizada por Paul Spicker (2007), que clasifica doce definiciones de pobreza agrupadas de acuerdo con las condiciones materiales (destacándose la limitación de recursos, las necesidades y el patrón de privaciones), con base en las condiciones sociales (la pobreza, en este sentido, estaría asociada a la ausencia de titularidades, ${ }^{1}$ a la carencia de seguridad básica, a la exclusión, a la dependencia y a la pertenencia a determinada clase social) y también en lo relativo a condiciones económicas (nivel de vida, desigualdad y posición económica).

Todas estas perspectivas conceptuales parecen compartir, asimismo, un presupuesto moral que remite a una privación inaceptable, lo que plantea

1 Entitlements, en el sentido de derechos a acceder a determinadas capacidades. 
de manera inevitable el debate entre pobreza absoluta y pobreza relativa. Amartya Sen (1992) fue uno de los más importantes proponentes de una concepción absoluta de la pobreza, basada en un "núcleo irreductible" de privaciones (esencialmente alimenticias, que, en definitiva, impiden el desarrollo de las capacidades), para luego incorporar en su teoría (solo en términos complementarios) a la pobreza relativa, asociada a los bienes y sus características. Peter Townsend (1979), por otro lado, se posiciona como un destacado referente teórico de la concepción de privación relativa en su definición de pobreza:

“[...] A su entender las personas, los hogares y los grupos de población son pobres si carecen de recursos para obtener los tipos de dieta, participar en las actividades y tener las condiciones de vida y las comodidades que se acostumbran en las sociedades de las cuales forman parte, es decir, sus recursos no les permiten tener acceso a los patrones ordinarios de vida, costumbres y actividades del individuo o la familia promedio de la sociedad en cuestión." (Townsend, 1979. Citado en Mora, 2010, p. 4).

Del mismo modo surge la discusión entre recursos y necesidades ${ }^{2}$ como formas de abordar conceptualmente la pobreza. Este debate parece ser ineludible para entender cómo se configuran las dinámicas de la pobreza:

"Las necesidades y los recursos para satisfacerlas cambian a lo largo del ciclo de vida de un hogar. Por ejemplo, cuando los niños son pequeños y numerosos, las necesidades son muchas, pero la capacidad de generar ingresos es baja, por lo que muchos hogares se sitúan por debajo de los umbrales de pobreza durante este período. Tanto el factor riesgo como la relación cambiante entre recursos y necesidades a lo largo del ciclo de vida pueden ser causas de pobreza temporal, aunque la segunda es de naturaleza estructural y la primera contingente." (Boltvinik, 2001, p. 38).

El presente artículo tiene como objetivo realizar un recorrido por los principales conceptosy taxonomías de los doscuerpos teóricos contemporáneos más relevantes en la elaboración del concepto multidimensional de la pobreza: el enfoque de las capacidades y la teoría de las necesidades humanas. En este sentido, también se incursiona por la génesis contextual de ambos cuerpos teóricos y, de forma lateral, en su ruptura o encuadre con el liberalismo económico y el liberalismo político.

2 La distinción entre recursos y necesidades no solo es necesaria para entender las dinámicas de la pobreza o para elegir una metodología de medición adecuada, sino que los determinantes de la pobreza son diferenciales con base en los citados enfoques (Fernández, Borrás y Ezquerra, 2017). 
Para cumplir dicho objetivo, se desarrollan los conceptos nodales del enfoque de las capacidades, destacando la relevancia de los funcionamientos de los bienes, sus características y su relación con el desarrollo de las capacidades humanas. Para poder llegar a esta instancia es menester aproximarse al origen contextual del enfoque de las capacidades, en la medida en que en la crítica a la noción de igualdad de la economía utilitarista se cimienta la conceptualización de Amartya Sen (1987). En una segunda instancia, se introduce el aporte de Martha Nussbaum (2000), que, enmarcándose en el enfoque de las capacidades, incorpora el gran debe de su fundador: una taxonomía de capacidades, de carácter universal y transcultural.

En lo que respecta a la teoría de las necesidades humanas, de Doyal y Gough (1994), son examinados los conceptos de necesidades básicas y necesidades intermedias, que se transforman en los elementos constitutivos de su taxonomía de satisfactores (ampliamente utilizada en la constitución del concepto de pobreza multidimensional contemporáneo). Finalmente, se examina la conceptualización de Max Neef y sus colaboradores (1993), destacando la propuesta sobre las necesidades humanas fundamentales y la distinción entre satisfactores violadores/destructores, inhibidores, sinérgicos y singulares.

\section{El enfoque de las capacidades, de Amartya Sen}

La génesis contextual del enfoque de las capacidades desarrollado por Sen (1979) se sitúa en la discusión con la noción de igualdad en la economía utilitarista y en la teoría rawlsiana de la justicia. ${ }^{3}$ Con importantes matices, estas perspectivas tienden a ignorar el funcionamiento de los bienes para los individuos, focalizándose en la utilidad que obtienen de ellos y desconociendo sus propiedades objetivas. Es así que, a fines de la década de los setenta, Sen propone la noción de igualdad de capacidad básica como superadora de las serias limitaciones que sustentaban las perspectivas citadas.

El gran aporte de Sen (1987) en su crítica a la economía utilitarista se da en dos dimensiones. Por un lado, sin despegarse del liberalismo económico, plantea que las utilidades que los bienes tienen para las personas pueden ser comparadas y no solo medidas. Por lo tanto, se puede elaborar un ranking de utilidades pensando en ellas como factores en la evaluación del bienestar humano. Esto hubiera sido impensable para la economía neoclásica en la medida en que cualquier mirada exterior ${ }^{4}$ (en adelante, $\mathrm{K}$ ) que evaluara la utilidad individual violaría los principios de libre elección de los bienes, ya

3 En el presente artículo el análisis se centra en la crítica a la economía utilitarista, en la medida en que la conceptualización de Sen se desarrolla a partir de la discusión con ella.

4 En la bibliografía especializada las referencias a esta mirada exterior se hacen con la nomenclatura $\mathrm{K}$. 
que el individuo sería el único juez legítimo para seguir las metas que se proponga. De ahí que el umbral ${ }^{5}$ (en adelante, $\mathrm{Z}$ ) de utilidad sea diferente en cada caso para el enfoque utilitarista. En segundo lugar, Sen critica al enfoque utilitarista por no tener en cuenta las características de los bienes ni el funcionamiento que estos tienen para las personas, que incluso trasciende la propia concepción material. La limitación del utilitarismo al desconocer los logros sociales que van más allá del bienestar individual ${ }^{6}$ se anclarían en una ontología egoísta (base del homo economicus) que olvida el comportamiento humano efectivo (San José, 1999).

La noción de libertad en sentido positivo es central en Sen y tiene mucho que ver con el concepto de capacidades (capabilities), pero es conveniente adentrarse aún más en el calado conceptual del enfoque de funcionamientos para finalmente volver a la noción de capacidades. Sen dirá que la mera posesión de bienes (commodities) por parte de las personas no basta para evaluar el impacto que estos tienen en su bienestar y en cómo contribuyen a su felicidad. En este sentido, cita como ejemplo el caso de una persona portadora de una patología parasitaria: ${ }^{7}$ su consumo de una cierta cantidad de alimentos seguramente resultará insuficiente, mientras que una persona sana tendrá más que satisfecha su necesidad nutricional con la misma ración. Para superar esta limitación conceptual propia de la idea de igualdad que sustenta el utilitarismo, Sen introduce dos conceptos clave. En primer lugar, introduce a las características $^{8}$ (characteristics) como aquellas propiedades deseables de los commodities, que, sin embargo, tampoco brindan información sustantiva sobre lo que las personas pueden hacer efectivamente con ellas.

Por lo tanto, en una segunda instancia propone a los funcionamientos (functionings) de los bienes como los logros obtenidos por las personas a partir del uso de un determinado bien. El funcionamiento sería, de esta manera, la instancia intermedia en términos temporales entre los bienes y la utilidad (planteada en términos de felicidad). De modo que Sen propone un enfoque de evaluación del bienestar basado en los funcionamientos que supera ampliamente al utilitarista, ya que tiene en cuenta las características de los bienes y es capaz de comparar las mediciones del bienestar de las

5 En los estudios de pobreza suele denominarse a los umbrales de medición con la letra Z (Fernández, 2010).

6 En el planteamiento de Sen (1987) existen dos grandes visiones en torno a los intereses de las personas. Estas radican en el "bienestar" y la "ventaja": mientras que el bienestar está relacionado con los logros de una persona y en cómo estos resultan en mayor calidad de vida y felicidad, la ventaja está asociada con las oportunidades y tendría un mayor potencial explicativo para el bienestar que el bienestar en sí mismo, ya que en el acceso a las oportunidades se encuentra la capacidad de agencia de las personas.

$7 \quad \mathrm{Al}$ ejemplo citado por el autor se podrían agregar los casos de los celíacos, los diabéticos o las embarazadas, entre otros.

8 Las características de los bienes podrían ser, a modo de ejemplo, los valores nutricionales de los alimentos. 
personas desde $\mathrm{K}$, así como también de establecer un $\mathrm{Z}$ basado en los funcionamientos y la libertad necesaria para el desarrollo de las capacidades, al mismo tiempo que mantiene la premisa liberal de que los individuos tienen capacidad de agencia para elegir qué vida quieren vivir, qué bienes comprar, qué parejas tener y qué hacer en sus horas de descanso, por nombrar algunos de los funcionamientos posibles. Asimismo, este razonamiento permite conceptualizar a la pobreza de un modo universal/absoluto en cuanto al acceso a las capacidades y los funcionamientos derivados de ellas, y fundar un concepto relativo de la pobreza en lo que tiene que ver con el acceso a los diferentes bienes y sus características, que varían de acuerdo con las diferentes culturas y momentos históricos.

De esta forma, Sen plantea matemáticamente el enfoque de los funcionamientos con las siguientes ecuaciones:

$$
\text { 1) } b_{i}=f_{i}\left(c\left(x_{i}\right)\right)
$$

En esta ecuación, $x$ representa los bienes (commodities) que la persona $i$ posee, $c$ es la función de las características de los bienes y el vector $f$ representa a los funcionamientos de determinada persona $i$ (el patrón de uso de los bienes, qué puede hacer esa determinada persona con ellos y cuáles son sus logros). Finalmente, y como resultado, el vector $b i$ refiere a los funcionamientos alcanzados por las personas.

Esto da lugar a la ecuación de felicidad, siempre entendida en relación con los funcionamientos:

$$
\text { 2) } u_{i}=h_{i}\left(f_{i}\left(c\left(x_{i}\right)\right)\right.
$$

En la presente ecuación, se agregan los vectores $h$ y $u$, siendo $h$ la función de felicidad relacionada con los funcionamientos logrados por las personas $i$, mientras que la función $u$ es la evaluación del bienestar (utilidad) de las personas con base en el producto de funcionamientos y felicidad de las personas $i$.

Planteado de esta manera el enfoque de funcionamientos, toca definir las capacidades $(Q)$ como la libertad que tienen las personas de adquirir los bienes $x$ con determinadas características $c$ y de optar por una serie de funcionamientos $f$ que deriven en logros y bienestar en sus vidas. Es así que la libertad positiva tiene que ver con las oportunidades de las que disponen las personas para su desarrollo pleno y el derecho a decidir sobre sus bienes, en su capacidad de agencia. Matemáticamente, la ecuación que incluye a las capacidades y a las titularidades (entitlements, en el sentido de derechos a acceder a determinadas capacidades, representados por $X$ ) y al conjunto de funcionamientos $F$ elegidos por las personas $i$ se desarrolla de la siguiente forma:

3) $\mathrm{Q}_{\mathrm{i}}\left(\mathrm{X}_{\mathrm{i}}\right)=\left[\mathrm{b}_{\mathrm{i}} \mid \mathrm{b}_{\mathrm{i}}=\mathrm{f}_{\mathrm{i}}\left(\mathrm{c}\left(\mathrm{x}_{\mathrm{i}}\right)\right)\right.$ para algunos $f_{i}(\cdot) \in F_{i}$, y para algunos $\left.x_{i} \in X_{i}\right]$

En la coronación de la obra de Sen, se destaca la noción de libertad positiva y también se pone de relevancia un tipo de racionalidad que tiene 
que ver con el comportamiento humano efectivo basado en la ética y que trasciende la racionalidad utilitarista basada en la maximización de beneficios.

\section{Las capacidades funcionales centrales: el aporte de Nussbaum}

Uno de los reproches conceptuales que se le han formulado a Sen tiene que ver con la no elaboración de una lista o un set de capacidades y funcionamientos. La filósofa estadounidense Martha Nussbaum (2000) desarrolla en su obra esta crítica a Sen, además de poner en el tapete la cuestión de las desigualdades de género, haciendo énfasis en la precaria situación de las mujeres de los países en desarrollo (tomando como referencia a India) en las postrimerías del siglo XX. De este forma, Nussbaum, quien se define como una pensadora neoaristotélica y feminista, comparte el concepto de capacidades y funcionamientos formulado por Sen, pero va aún más lejos, en busca de una legitimación legal de las capacidades en la construcción de un universalismo transcultural que tenga en cuenta la dignidad humana y la noción aristotélica de ser en acto y ser en potencia, en cualquier sociedad y cultura.

Nussbaum propone que las capacidades y los funcionamientos deberían ser parte del orden constitucional de los Estados nacionales, al mismo tiempo en que hace hincapié en el umbral de satisfacción de estas capacidades, para lo cual desarrolla una lista de "capacidades funcionales humanas centrales", 9 concebida como una taxonomía universal, aunque abierta a cambios sujetos a la especificidad histórica de las distintas sociedades y circunstancias locales.

El set propuesto por Nussbaum consiste en las siguientes capacidades:

1) la vida: todas las personas tienen derecho a vivir temporalmente una vida normal, es decir, no morir prematuramente y vivir una vida digna de ser vivida;

2) salud corporal: también tienen derecho a ser capaces de tener buena salud, incluyendo la reproductiva, y a tener una alimentación y vivienda adecuadas;

9 La autora distingue tres tipos de capacidades, entre las que incluye a las básicas, las internas y las combinadas. Las capacidades básicas consisten en aquellas referentes al goce de salud corporal, base necesaria para poder llevar adelante funcionamientos: poder ver y poder oír. Volviendo a sus raíces aristotélicas, Nussbaum dice que: "A newborn child has, in this sense, the capability for speech and language, the capability for love and gratitude, the capability for practical reason, the capacity for work" (Nussbaum, 2000, p. 84). Las capacidades internas, por otro lado, refieren al estado de desarrollo de la persona misma, en los requerimientos personales propios para el desarrollo de funcionamientos. Finalmente, las capacidades combinadas son aquellas que integran las capacidades básicas como las premisas físicas y materiales mínimas necesarias para el desarrollo del funcionamiento, así como también las capacidades internas de madurez y desarrollo propicios para dicho funcionamiento. La lista de capacidades centrales consistiría entonces en capacidades combinadas y la autora argumenta que esto es una síntesis de las circunstancias materiales y sociales de las personas. 
3) integridad corporal: refiere a libertad de movimiento, en el entendido de que las personas son soberanas de su cuerpo; aquí se hace hincapié en la protección contra el abuso, incluyendo el infantil y sexual, contra la violencia doméstica y los asaltos, así como a las oportunidades para la satisfacción sexual y la reproducción;

4) sentidos, imaginación y pensamiento: dentro de esta dimensión, Nussbaum propone la capacidad que hace vivir una vida "verdaderamente humana": desde la alfabetización y el acceso a un sistema educativo capaz de desarrollar el pensamiento crítico en las personas y la autoexpresión, hasta la libertad de expresión y de culto;

5) emociones: capacidad referida a poder amar y cuidar a otras personas y a tener un desarrollo emocional sin impactos provocados por la ansiedad o el miedo, fruto de abusos o negligencia;

6) razón práctica: ser capaz de formar una concepción del bien y de planificar la propia vida;

7) afiliación: la autora propone una división en lo referente a la presente dimensión: por un lado, destaca la importancia del reconocimiento social y la empatía entre la persona y los otros, resaltando a la amistad como una de sus instancias clave, y, por otro lado, enfatiza la protección contra discriminaciones por raza, sexo, religión, casta, etnia u origen nacional. Asimismo, la afiliación tiene que ver con el acceso a un trabajo digno, a la vez que implica el desarrollo de una razón práctica sobre el plan de vida de la persona, la autoestima y la no humillación;

8) otras especies: tener en cuenta, respetar y proteger la fauna y flora y el mundo de la naturaleza en general;

9) jugar: disfrutar de actividades recreativas, poder reír;

10) control sobre el entorno de uno: el entorno de las personas es presentado en una faceta política, que comprende el acceso a la participación política y protecciones a la libertad de expresión y asociación, y una faceta material, que versa sobre el derecho a tener bienes, tierra y empleo, todo en igualdad de condiciones con respecto a las demás personas y no solo formalmente, sino en términos de oportunidades reales (Nussbaum, 2000).

Cabe señalar varias cuestiones respecto a la lista de capacidades funcionales humanas centrales propuesta por Nussbaum. En primer lugar, se destaca la novedosa incorporación de los derechos y garantías necesarias para el desarrollo personal de mujeres y niños, que, en consonancia con los Objetivos del Milenio, ${ }^{10}$ persigue el objetivo de empoderar a las mujeres

10 En el año 2000, la Asamblea General de la Organización de las Naciones Unidas aprobó una declaración con base en una serie de objetivos comunes a todos los países, entre los que destaca la necesidad de consolidar la igualdad entre varones y mujeres, posicionando en la agenda pública global las desigualdades de género (ONU, 2000). 
y discutir desde una perspectiva feminista sus roles tradicionales. Por otro lado, también es destacable la preocupación por el medioambiente, las otras especies del mundo animal y las capacidades referidas a funcionamientos que exceden las características de bienes/recursos materiales: el cultivo de las emociones y la razón práctica, así como la necesaria participación en la vida política de las sociedades. Nussbaum rescata del marxismo la necesidad de oportunidades reales en el plano de las condiciones materiales de existencia, en detrimento de una mera formalidad legal y burguesa, pero esto no tiene ningún tipo de concesión en su visión liberal sobre las garantías constitucionales de la democracia y la libertad política: en otras palabras, el bienestar material no podría estar por encima de la libertad individual y política de las personas.

\section{La teoría de las necesidades humanas}

La génesis contextual en que se desarrolla la teoría de las necesidades humanas de Len Doyal y Ian Gough (1994) está signada por la consolidación y predominancia de políticas neoliberales impulsadas por el ascenso de lo que los autores denominan la "nueva derecha", a principios de la década del ochenta. Esto habría signado un paisaje marcado por la decadencia de los Estados de bienestar y las políticas redistribucionistas en Europa occidental. La propuesta desarrollada por Doyal y Gough es presentada como una alternativa al neoliberalismo y conservadurismo político. La marcada impronta neokantiana de los autores, por otro lado, se ve reflejada en su intención de dar universalidad y objetividad a las necesidades humanas básicas, además de reconocer que son constructos sociohistóricos, rechazando las visiones naturalistas (defendidas por el utilitarismo y las políticas neoliberales), relativistas y culturalistas de la necesidad. A diferencia del enfoque de capacidades propuesto por Sen, la obra de Doyal y Gough rompe con los preceptos del liberalismo económico, estableciendo necesidades universales y objetivas y un rol preponderante del Estado en las políticas públicas. Esta ruptura, que se aleja del enfoque de las capacidades, logra encuadrarse en el liberalismo político por su férrea defensa del sistema democrático y republicano. En este sentido, se asemeja más a la versión de las capacidades humanas centrales propuesta por Nussbaum.

Los aportes realizados por la teoría de necesidades humanas de Doyal y Goughsonfundamentalesenlaconstruccióndelaperspectivamultidimensional contemporánea sobre la pobreza. Estos teóricos argumentan que existen dos tipos de necesidades humanas básicas de características universales y objetivas: por un lado, la necesidad concerniente a la supervivencia y a la salud física y, por otro, aquella relativa a la autonomía individual, convirtiéndose en condiciones sine qua non de toda acción individual para cualquier cultura o sociedad y posibilitando la participación efectiva en la vida social con el 
fin de lograr cualquier objetivo que se considere valioso. La satisfacción de estas necesidades básicas es un prerrequisito fundamental para el desarrollo personal de los individuos en el marco de la participación en la vida social de cada cultura y sociedad:

"Toda nuestra vida, aun cuando estemos solos, está dominada por lo que aprendemos de otros, por cómo valoran lo que creen que hemos aprendido y cómo reaccionan a los cambios de nuestras acciones sobre la base de dicha valoración. En otras palabras, desarrollamos una concepción propia de quiénes somos a través del descubrimiento de lo que somos o no capaces de hacer, un logro que se basa en nuestra participación en la vida social." (Doyal y Gough, 1994, p. 79).

Al igual que Nussbaum, Doyal y Gough le reprochan a Sen el no haber elaborado una lista sistematizada sobre las capacidades y los funcionamientos correspondientes, conceptos que los autores dicen compartir, y, en este sentido, pretenden incorporar el enfoque de Amartya Sen en su propia teoría. Para esto, desarrollan los conceptos de satisfactores y necesidades intermedias, de manera de poder "operacionalizar" las mencionadas necesidades básicas de salud física y autonomía individual. De esta forma, se refieren a las características de satisfactores universales como necesidades intermedias:

“[...] pueden así definirse como aquellas cualidades de los bienes, servicios, actividades y relaciones que favorecen la salud física y la autonomía humanas en todas las culturas. Por ejemplo, el aporte diario de calorías para un grupo determinado de personas constituye una característica de casi todos los productos alimenticios cuya relevancia es común a todas las culturas. [...] La categoría de características de satisfactores universales tiende así el puente crítico entre las necesidades básicas universales y los satisfactores relacionados con lo social.” (Doyal y Gough, 1994, pp. 201, 202).

Esta apreciación conceptual tiene una cierta analogía con el enfoque de funcionamientos en Sen, que era ubicado como la instancia temporalmente intermedia entre el uso de los bienes y sus características y la utilidad entendida en términos de felicidad a partir de los funcionamientos. En la constitución de la taxonomía de necesidades intermedias elaborada por Doyal y Gough, la necesidad básica de supervivencia y salud física se propone en un formato de satisfactores universales planteados en términos negativos (siendo la privación de dichas necesidades una carencia inaceptable): 1) alimentos nutritivos y agua limpia; 2) alojamientos adecuados para la protección; 3) ambiente laboral desprovisto de riesgos; 4) medio físico desprovisto de riesgos; y 5) atención sanitaria apropiada. Por otro lado, las necesidades intermedias referidas a la necesidad básica de autonomía individual se distribuyen de la siguiente manera: 6) seguridad de la infancia; 7) relaciones primarias significativas; 8) seguridad 
física; 9) seguridad económica; 10) enseñanza adecuada; y 11) seguridad en el control de nacimientos y en el embarazo y parto (Doyal y Gough, 1994). Los autores remarcan, al igual que Martha Nussbaum, la inclusión de la necesidad intermedia número 11 como el reconocimiento a las especificidades biológicas de las mujeres, en el marco de un necesario empoderamiento desde las políticas sociales dirigidas al alivio de la pobreza.

La legitimación ${ }^{11}$ de las necesidades humanas básicas y sus satisfactores y necesidades intermedias descansa en el modelo biomédico y en los derechos humanos, respectivamente. De este modo, Doyal y Gough también adhieren al liberalismo político, en cuanto a que consideran que debe existir necesariamente una serie de premisas políticas para el correcto desarrollo de las personas y para la optimización de la satisfacción de las necesidades básicas. Desde el liberalismo económico, se podrían realizar fuertes críticas a la teoría de las necesidades humanas, en la medida en que la presencia de $\mathrm{K}$ determinando las necesidades humanas básicas y sus satisfactores sería, en el mejor de los casos, una violación flagrante a la libre elección de los individuos.

Finalmente, otra de las coincidencias de la conceptualización de Doyal y Gough con la teoría de Sen tiene que ver con el debate entre pobreza absoluta y pobreza relativa. Para estos autores, las necesidades intermedias tomarían una forma universal, mientras que sus satisfactores serían históricamente variables, es decir, relativos. A modo de ejemplo, las necesidades intermedias que implican la alimentación y el alojamiento serían comunes a todos los pueblos, pero las formas de satisfacerlas serían diferentes en cada sociedad debido a la inconmensurable diversidad de métodos gastronómicos y arquitectura de refugios contra las inclemencias de la naturaleza.

\section{El aporte latinoamericano a la teoría de necesidades humanas}

En América Latina, destaca el enfoque propuesto por Max Neef, Elizalde y Hopenhaym (1993), que posiciona a las necesidades humanas como elemento central en el desarrollo de las personas y los países. El contexto sociohistórico que signa esta producción intelectual está marcado por la predominancia de los modelos económicos neoliberales y el fracaso de las políticas estatalistas que llegaron a ser hegemónicas a mediados del siglo XX en gran parte de América Latina.

De manera análoga a la argumentación de Doyal y Gough, Max Neef y sus colaboradores plantean la necesidad de elaborar mediciones del bienestar y de la pobreza que tengan en cuenta a las personas y no a los objetos, poniendo como ejemplo las limitaciones del producto interno bruto (PIB)

11 Para los autores, las ciencias naturales y las ciencias sociales juegan un rol destacado en la constitución y legitimación de las necesidades humanas. 
como indicador social y de calidad de vida. Otra de las coincidencias de Max Neef y su equipo con los enfoques antes presentados tiene que ver con la distinción entre las "necesidades humanas fundamentales" y sus satisfactores. $\mathrm{El}$ argumento en este sentido es que mientras las primeras adquieren una significación universal, objetiva y que aplica a todos los períodos históricos, los satisfactores son relativos y varían según las diferentes culturas. Las necesidades humanas son clasificadas por estos autores de la siguiente forma:

"En este documento se combinan dos criterios posibles de desagregación: según categorías existenciales y según categorías axiológicas. Esta combinación permite operar con una clasificación que incluye, por una parte, las necesidades de Ser, Tener, Hacer y Estar; y, por la otra, las necesidades de Subsistencia, Protección, Afecto, Entendimiento, Participación, Ocio, Creación, Identidad y Libertad." (Max Neef, Elizalde y Hopenhaym, 1993, p. 37).

Asimismo, los autores prefieren hablar de "pobrezas" y no de pobreza, a la que consideran atada a un principio economicista de asignación de recursos. Las pobrezas se dan en aquellas categorías de necesidades axiológicas descritas antes, donde no existe una satisfacción adecuada, produciéndose así una serie de "patologías" sociales que los autores identifican como desempleo, hiperinflación, patologías políticas (por ejemplo, la falta de libertad de expresión), miedo, violencia, marginación y exilio. Las necesidades humanas fundamentales no solo deben ser abordadas por un enfoque de carencias, sino también desde la potencialidad que encierran, transformándolas, en una construcción dialéctica entre carencia y potencialidad, potenciando de esta manera, valga la redundancia, la subjetividad y el desarrollo individual y colectivo.

La matriz ${ }^{12}$ de necesidades y satisfactores propuesta por Max Neef, Elizalde y Hopenhaym muestra muchas semejanzas con la elaborada por Doyal y Gough y con la lista de capacidades funcionales humanas centrales de Martha Nussbaum. Asimismo, proponen formas no deseadas de satisfacción de necesidades, que incluyen supuestos satisfactores "violadores o destructores", por ejemplo, el armamentismo, que pretende satisfacer la necesidad de protección, pero que, en definitiva, niega la posibilidad de satisfacer las necesidades de subsistencia, afecto, participación y libertad. En este sentido, también se destaca la existencia de "pseudosatisfactores", un ejemplo de los cuales sería la explotación

12 Debido a que la matriz taxonómica de necesidades y satisfactores elaborada por Manfred Max Neef y sus colaboradores es extensa, se enumeran en el presente trabajo los tipos de necesidades humanas fundamentales, entendiendo que los satisfactores más comunes propuestos por el autor han sido mencionados a lo largo del trabajo: desde la alimentación, la vivienda adecuada, el acceso a la educación y a la salud hasta la libre participación en la vida social y política. Por razones de espacio, no se desarrolla de manera exhaustiva la citada matriz (disponible en Max Neef, Elizalde y Hopenhaym, 1993, pp. 53, 54). 
desmedida de recursos naturales, que aparenta satisfacer la necesidad de subsistencia. Una tercera categoría de satisfactores no deseados incluye a los satisfactores "inhibidores", que "[...] salvo excepciones, se hallan ritualizados, en el sentido de que suelen emanar de hábitos arraigados" (Max Neef, Elizalde y Hopenhaym, 1993, p. 58). Los ejemplos ofrecidos en este sentido oscilan entre familias sobreprotectoras, producción de tipo taylorista y aulas autoritarias. Finalmente, los autores mencionan a los satisfactores "singulares", que, por sus atributos, buscan satisfacer una necesidad a la vez. En sus formas institucionales, estos satisfactores serían el voto (participación), los programas asistenciales de vivienda (subsistencia) o el ejército profesional (protección). Para solucionar los problemas que encierra este tipo de satisfactores, los autores proponen una serie de satisfactores "sinérgicos", a los que reconocen como contrahegemónicos pero que, en su afán de satisfacer una necesidad determinada, contribuyen a la satisfacción simultánea de las demás. En el presente trabajo se quiere destacar a la lactancia materna, la producción autogestionada, la educación popular, la existencia de organizaciones comunitarias democráticas y la democracia directa como algunos de los satisfactores sinérgicos más relevantes.

\section{Conclusiones}

Este artículo intentó hacer un repaso de las teorías que fundamentan un concepto multidimensional de la pobreza, que se torna absoluto en el espacio de las capacidades/funcionamientos/necesidades y relativo en lo concerniente a bienes/satisfactores. Como se ha planteado durante el trabajo, las diferencias más agudas entre los enfoques de capacidades y funcionamientos y el de la teoría de las necesidades humanas radican en la tesitura más o menos liberal de dichas perspectivas. Aunque en las dos visiones existe una declarada adhesión al liberalismo en sus diferentes formas, Amartya Sen logra elaborar un ranking de las utilidades, con base en su enfoque de los funcionamientos, que el utilitarismo ignoraba debido a su desconocimiento de las características de los bienes. De esta manera, el K propuesto por Sen no viola los principios liberales de la libre elección individual de vivir y ser feliz, al mismo tiempo que reconoce un núcleo irreductible de privaciones asociado a requerimientos nutricionales. El enfoque de la teoría de las necesidades humanas es netamente liberal en lo político, pero no desde el punto de vista económico: Doyal y Gough proponen un K decididamente ajeno a las elecciones individuales, basado en una legitimación biomédica y legal de la salud física y la autonomía individual, respectivamente. Su concepción de pobreza es, al igual que en el enfoque de los funcionamientos, absoluta en lo concerniente a las necesidades, pero relativa en cuanto a los satisfactores. 
Otros puntos a destacar son las contribuciones que han hecho las diferentes perspectivas de acuerdo con su contexto histórico. El enfoque de las capacidades/funcionamientos desarrollado por Sen en las décadas siguientes a la segunda posguerra pone en entredicho las bondades del PIB como indicador de bienestar social, planteando la ambiciosa construcción de un $\mathrm{Z}$ capaz de medir y comparar el bienestar del individuo. A la postre, este avance conceptual llevó nada más y nada menos que a la elaboración del índice de desarrollo humano (IDH), que ha marcado sin dudas un clivaje en la historia de la medición de bienestar de los países, dando lugar a la elaboración de políticas públicas más consistentes con las diferentes realidades. Martha Nussbaum, por su parte, retoma el planteamiento de Sen desde una mirada feminista que pone en el centro de la cuestión social a las mujeres y los niños, haciendo énfasis en los países en desarrollo, lo que marca el comienzo de una nueva era en las postrimerías del siglo XX.

Finalmente, Doyal y Gough, en pleno colapso del bloque comunista en Europa oriental y sin deslindarse del liberalismo político, plantean un papel estatal relevante en la economía y en la vida social, en el marco de un auge global de políticas promercado y de una reorganización geopolítica que consolidaba a las democracias liberales occidentales como las hegemónicas portadoras de legitimidad, con una profunda crisis de los modelos de bienestar socialdemócratas. En esta sintonía, Max Neef y sus colaboradores reconocen el fracaso de las políticas estatalistas y neoliberales en América Latina, proponiendo el modelo de desarrollo a escala humana como alternativa.

\section{Referencias bibliográficas}

Boltvinik, J. (2001). Métodos de medición de la pobreza. Concepto y tipología. En L. Gallardo; J. Osorio y M. Gendreu (2001). Los rostros de la pobreza. Tomo III. México: Limusa.

Doyal, L. e I. Gough (1994). Teoría de las necesidades humanas. Barcelona: Economía crítica.

Fernández, T. (2010). Hacia un enfoque multidimensional de la pobreza: cuestiones teóricas. En: M. Serna (coord.) (2010). Pobreza y (des) igualdad en Uruguay: una relación en debate. Montevideo: CLACSOFacultad de Ciencias Sociales, UDELAR, pp. 171-192. Disponible en: $<$ http://biblioteca.clacso.edu.ar/gsdl/collect/clacso/index/assoc/D2067.dir/ pobrezaydesigualdadenuruguay.pdf $>$ [acceso 30 de junio de 2020]. 
Fernández, T.; V. Borrás y P. Ezquerra (2017). Pobreza de ingresos y pobreza multidimensional. Una comparación de sus determinantes para Uruguay en 2015. Revista Sociedad, 37, pp. 155-186. Disponible en: <https:// publicaciones.sociales.uba.ar/index.php/revistasociedad/article/ view/2977/2466> [acceso 30 de junio de 2020].

Max Neef, M.; a. Elizalde y M. Hopenhaym (1993). Desarrollo a escala humana. Conceptos, aplicaciones y algunas reflexiones. Montevideo: NordanComunidad.

Mora, M. (2010). La multidimensionalidad y la heterogeneidad de la pobreza: desafios analíticos para la sociología [Mimeo]. México: El Colegio de México.

Nussbaum, M. (2000). Women and human development. The capabilities approach. Cambridge: Cambridge University Press.

ONU (2000). Objetivos de Desarrollo del Milenio [en línea]. Disponible en: $<$ https://www.un.org/millenniumgoals/> [acceso 30 de junio de 2020].

San José, A. (1999). Crítica de Amartya Sen a la economía utilitarista. Cuadernos de anuario filosófico. Pamplona: Servicio de publicaciones de la Universidad de Navarra. Disponible en: < https://dadun.unav.edu/ bitstream/10171/6090/1/86.pdf $>$ [acceso 30 de junio de 2020].

Sen, A. (1979). Equality of What? Stanford: Stanford University.

Sen,A. (1987). Commodities and capabilities. Nueva Delhi: Oxford India Paperbacks.

Sen, A. (1992). Sobre conceptos y medidas de pobreza. Comercio Exterior, 42(4), pp. 310-322.

Spicker, P. (2007). Definiciones de pobreza: doce grupos de significados. En: P. Spicker; S. Álvarez Leguizamón y D. Gordon (eds.) (2007). Pobreza. Un glosario internacional. Buenos Aires: CLACSO, pp. 291-306. Disponible en: <http://biblioteca.clacso.edu.ar/gsdl/collect/clacso/index/assoc/D9376. dir/06spicker.pdf $>$ [acceso 30 de junio de 2020].

\section{Contribución de autoría}

Este trabajo fue realizado en su totalidad por Diego Amarilla. 